\title{
The $P$ wave duration in patients with atrial fibrillation undergoing cryoballoon pulmonary vein isolation. Preliminary results
}

\author{
Jacek Marcin Zawadzki ${ }^{1}$, Jakub Adamowicz ${ }^{2}$, Agnieszka Sławuta ${ }^{2}$, \\ Aleksandra Gajek ${ }^{1}$, Dorota Zyśko', Jacek Gajek ${ }^{1,2}$
}

\author{
${ }^{1}$ Wrocław Medical University, Wrocław, Poland \\ ${ }^{2}$ Kłodzko County Hospital, Department of Cardiology, Kłodzko, Poland
}

\begin{abstract}
The pulmonary vein isolation remains the major target of atrial fibrillation ablation. The cryoablation lesions in the left atrium are supposed to disconnect the pulmonary vein from the atrium on the atrial side of the orifices. We hypothesized that the cryoballoon pulmonary vein isolation could result in the prolongation of the $\mathrm{P}$ wave duration. The aim of the study was to assess the duration of the $P$ wave in 12-lead electrocardiogram and the influence of pulmonary vein isolation on this parameter. The study group included 21 patients (11 women and 10 men) $66.2+/-7.4$ years of age undergoing cryoballoon ablation. In order to measure the $P$ wave duration, we used the constant acquisition of electrogram and the electrocardiographic channels provided by LABSYSTEM $^{\text {TM }}$ Pro EP Recording System (Boston Scientific), magnifying the leads 64x. We calculated the duration in the simultaneously recorded 12-lead ECG, from the beginning of the earliest recorded P wave deflection, until the end of the latest $P$-wave deflection recorded in any lead. The $P$ wave duration in the entire study group was $141.7+/-$ $12.5 \mathrm{~ms}$ before the ablation and increased significantly to $151.1+/-11.5 \mathrm{~ms}(\mathrm{p}<0.05)$ after the procedure. The respective values in women were $144.1+/-4.3$ vs. $156.0+/-4.7 \mathrm{~ms}(p<0.01)$ and $139.1+/-4.6$ vs. $145.6+/-4.5 \mathrm{~ms}$ in men $(p<0.05)$. The mean standard deviation of every single measurement considered separately was $4.4+/-$ $2.1 \mathrm{~ms}$ before the cryoablation and $4.6+/-1.8 \mathrm{~ms}$ after the procedure $(p=$ not significant (n.s).), indicating very good reproducibility of the measurements. We concluded, that cryoballoon pulmonary vein isolation leads to the prolongation of the measured $\mathrm{P}$ wave duration. It seemed to result from conduction disturbances created by cryoablation. The clinical significance of the observed changes remains unknown.

Keywords: $\mathrm{P}$ wave duration $-\mathrm{P}$ wave dispersion $\bullet$ cryoablation $\bullet$ atrial fibrillation $\bullet$ pulmonary veins isolation
\end{abstract}

\section{Citation}

Zawadzki JM, Adamowicz J, Sławuta A, Gajek A, Zyśko D, Gajek J. The P wave duration in patients with atrial fibrillation undergoing cryoballoon pulmonary vein isolation. Preliminary results. Eur J Transl Clin Med 2018;1(1):44-47.

DOI: $10.31373 /$ ejtcm/96253 


\section{Introduction}

Pulmonary vein isolation remains the major target of atrial fibrillation ablation [1]. The procedure disconnects the electrical activity of pulmonary veins from the myocardial sleeves entering the veins from the atrium, thus disrupting focal and reentrant arrhythmogenic mechanisms [2]. Another possible outcome of the procedure is the damage of the autonomic plexi functions (both sympathetic and parasympathetic), which could be of considerable importance in certain subgroups of patients [3]. Finally, the decrease of the atrial myocardium mass is an important factor for self-perpetuation of the atrial arrhythmia [4]. Atrial fibrillation has a wide spectrum of clinical presentations, therefore probably each mechanisms described above can be effective in a particular subset of patients.

The pulmonary vein isolation does not target the histological substrate in the atrial myocardium, but separates the peripheral parts of the atrial musculature, which should lead to more uniform atrial activation from the electrical point of view. This mechanical approach could explain the expectation that as the cardiac muscle mass diminishes, the procedure is bound to shorten the duration of the $P$ wave and lower its amplitude. Properly performed balloon cryoablation lesions in the left atrium are supposed to disconnect the vein from the atrium on the atrial side of the orifices. The dimensions of the balloon are standard, and the different sizes of the veins' ostia or even common trunks are targeted with one balloon. This could be the reason for causing various lesions in respect to size and location. To some extent, this could be related to the operator's skills but the main effect is still the same - similar lesions in different locations.

We hypothesized that the cryoballoon pulmonary veins isolation could result in prolongation of the atrial electrical activation, resulting in longer $\mathrm{P}$ wave duration at least in a significant percentage of patients undergoing the treatment of atrial fibrillation by this method.

\section{Purpose}

The aim of the study was to measure the duration of the $P$ wave in 12-lead electrocardiogram and to assess the influence of pulmonary vein isolation by cryoballoon ablation on this parameter.

\section{Materials and methods}

The study group included 21 patients ( 11 women and 10 men) $66.2+/-7.4$ years of age undergoing pulmonary vein isolation due to atrial fibrillation. The exclusion criteria included the presence of atrial fibrillation directly before the procedure, and the low $P$ wave amplitude which could negatively influence the assessment of $P$ wave onset and offset. We used the cryoballoon method (Arctic Front Advance by Medtronic) delivered into the left atrium by standard approach via the right femoral vein, and transseptal-fluoroscopy-guided puncture with steerable catheter (FlexCath Advance by Medtronic). The anatomy and morphology of the left atrium and the veins were assessed using fluoroscopy contrast medium in the left and right anterior oblique views for the left and right pulmonary veins respectively. The Achieve electrode (Medtronic) had been used as the guide wire for mapping pulmonary vein potentials before, during, and after cryoenergy applications. The cryoablation was performed according to the manufacturer's recommendations and was assessed as successful considering the disappearance of previously present pulmonary vein activity. The constant temperature of <-40 degrees Celsius lasted for more than 120 seconds, and the time of total energy application did not exceed 480 seconds.

In order to measure the $\mathrm{P}$ wave duration, we used the constant acquisition of electrogram and the electrocardiographic channels provided by LABSYSTEM ${ }^{\mathrm{TM}}$ Pro EP Recording System (Boston Scientific) and magnified the 12lead ECG 64x. This scale enabled the accurate assessment of the $P$ wave duration without the artifacts. We used the concept of the total atrial activation time - calculated in the simultaneously recorded 12-lead ECG, from the beginning of the earliest recorded $P$ wave deflection, until the end of the last P-wave deflection recorded in any lead as described previously by our group [5]. To assure the exact and unbiased $P$ wave assessment, the measurements were taken from the mean value of 5 cardiac cycles before and after the cryoablation by two independent operators blinded to clinical data.

\section{Statistical analysis}

The analyzed parameters are presented as the means and standard deviations. The categorical variables were showed as percentages. The statistical analysis of the differences in $\mathrm{P}$ wave duration was performed, using paired Student's T test. P value $<0.05$ was set as statistically significant.

\section{Results}

The clinical characteristics of the study group is presented in Table 1.

Table 1. Patient demographics and selected clinical data

\begin{tabular}{|l|c|}
\hline \multicolumn{1}{|c|}{} & $\begin{array}{c}\text { Study group } \\
\text { (21 } \mathbf{~ p t s )}\end{array}$ \\
\hline Age (years) & $66.2+/-7.4$ \\
\hline Sex (\% female) & 52.4 \\
\hline HR (byst. (mmHg) & $124.0+/-27.0$ \\
\hline EF (\%) & $75.3+/-17.7$ \\
\hline DM/IGF/IGT (\%) & $56.7+/-12.8$ \\
\hline HT (\%) & 14.3 \\
\hline
\end{tabular}


Table 2. The changes in $\mathbf{P}$ wave duration in relation to the cryoballoon ablation

\begin{tabular}{|l|c|c|c|}
\multicolumn{1}{|c}{} & $\begin{array}{c}\text { P wave duration } \\
\text { before }(\mathbf{m s})\end{array}$ & $\begin{array}{c}\text { P wave duration } \\
\text { after }(\mathbf{m s})\end{array}$ & P value \\
\hline Study group & $141.7+/-12.5$ & $151.1+/-11.5$ & $<0,05$ \\
\hline Women $(n=11)$ & $144.1+/-4.3$ & $156.0+/-4.7$ & $<0.01$ \\
\hline Men $(n=10)$ & $139.1+/-4,6$ & $145.6+/-4.5$ & $<0.05$ \\
\hline Standard Deviation of every single measurement & $4.4+/-2.1$ & $4.6+/-1.8$ & n.s. \\
\hline
\end{tabular}

The $P$ wave duration changes are presented in Table 2 .

Only in 2 patients the mean $\mathrm{P}$ wave duration after the procedure was shorter in comparison to the initial measurements: in 1 man by $1 \mathrm{~ms}$ and in 1 woman by $3 \mathrm{~ms}$.

The standard deviation of every single measurement of the $\mathrm{P}$ wave duration mean value, considered separately before and after the cryoablation was small in comparison to the mean, indicating the low dispersion of the measurements obtained by the two observers. This confirms the reliability of the methodology used.

\section{Discussion}

The main result of our study is the noted increase in $\mathrm{P}$ wave duration in patients undergoing cryoballoon pulmonary veins isolation. This parameter was not extensively studied yet.

The studies that evaluate the changes in $\mathrm{P}$ wave after different ablation approaches have provided inconsistent results. In a group of 27 patients Ogawa et al. observed a significant decrease of $P$ wave duration after cryoballoon ablation the subgroup without recurrence $(161+/-7$ to $151+/-8 \mathrm{~ms})$ and an insignificant change in the group with atrial fibrillation recurrence [6]. In 50 patients with paroxysmal atrial fibrillation Zhao et al. found the decrease in $P$ wave duration on surface ECG after ablation. This change however, was not related to arrhythmia recurrence [7]. In another study including 45 patients, a significant decrease of $P$ wave duration was found both after cryoballoon and radiofrequency ablation, but its correlation to arrhythmia recurrence was not assessed [8]. Kizilirmak et al. assessed the P wave duration in a group of 61 patients undergoing cryoballoon ablation of atrial fibrillation and found a decrease in this parameter - 109.7+/-18.4 at baseline to $91.4+/-22.5$, surprisingly lower in comparison with our data [9]. One explanation could be the difference in the mean age of the patients studied; our study group was $>10$ years older. On the other hand the methodology of the $P$ wave measurement used in that study was particularly prone to the noise/artifacts. Moreover, they assessed the so-called $\mathrm{P}$ wave dispersion. This could be the explanation for relatively high standard deviation values exceeding $20 \%$ of the mean value.
A couple of years ago our team published a paper introducing the concept of total atrial activation time and challenging the concept of so-called $\mathrm{P}$ wave dispersion. We conclusively demonstrated that the $P$ wave dispersion is actually a measurement artifact related to the imprecise measurement of $P$ wave. It does not address the nearly isoelectric parts of the $P$ wave at its onset and an end. If the measurement is done correctly, the differences between the longest and shortest $P$ wave are equal to zero [5]. This methodological issue, not relating to our terminology per se, has been already addressed in the literature [10].

The mechanism underlying the change of $P$ wave duration after ablation remains elusive. One of the suggested mechanisms for its shortening could be a interruption of the electrical connection between the veins and the atrium, leading to the shortening of the terminal portion of $P$ wave, which indicates late activation of PVs-LA region [11]. Our explanation of the prolongation of the $P$ wave duration after the procedure could be the creation of ablation lesions in the left atrial roof with prolonged activation of left high atrium. Indeed, the decreased number of depolarizing myocardial cells after ablation may influence the decrease of $P$ wave duration, however the conduction disturbances in the high left atrium would have a greater impact on this parameter, which might explain our results. As the prolongation of the $P$ wave duration is the dominant indicator of future atrial fibrillation recurrences, the main question of clinical significance of the observed changes remains [12].

\section{Limitation of the study}

The most significant limitation of our study is the relatively small number of the patients included. On the other hand, only in two cases the $P$ wave duration was shorter after the procedure and by very small time differences. The essential factor could be the operator's experience but on the other hand, the cryoballoon approach leaves a very narrow margin for technical differences among the particular operators.

\section{Conclusions}

Cryoballoon pulmonary vein isolation leads to the prolongation of the appropriately measured $P$ wave 
duration. It seems that the conduction disturbances created by cryoablation dominate over the decrease of atrial myocardium mass after the procedure. The clinical significance of the observed changes remains unknown.

\section{References}

1. Oral H, Knight BP, Tada H, Ozaydın M, Chugh A, Hassan S, et al. Pulmonary vein isolation for paroxysmal and persistent atrial fibrillation. Circulation. 2002;105(9):1077-81.

2. Haïssaguerre M, Shah DC, Jaïs P, Hocini M, Yamane T, Deisenhofer I, et al. Electrophysiological breakthroughs from the left atrium to the pulmonary veins. Circulation. 2000;102(20):2463-5.

3. Pappone C, Santinelli V, Manguso F, Vicedomini G, Gugliotta F, Augello G, et al. Pulmonary vein denervation enhances long-term benefit after circumferential ablation for paroxysmal atrial fibrillation. Circulation. 2004;109(3):327-34.

4. Mulukutla S, Althouse AD, Jain SK, Saba S. Increased left atrial size is associated with higher atrial fibrillation recurrence in patients treated with antiarrhythmic medications. Clin Cardiol. 2018;41(6):825-9.

5. Zimmer K, Przywara W, Zyśko D, Sławuta A, Gajek J. The nature of P-wave dispersion - A clinically useful parameter that does not exist. Int J Cardiol. 2016;212(1):59-60.

6. Ogawa M, Kumagai K, Vakulenko M, Yasuda T, Siegerman C, Garfinkel A, et al. Reduction of P-wave duration and successful pulmonary vein isolation in patients with atrial fibrillation. J Cardiovasc Electrophysiol. 2007;18(9):931-8.

7. Zhao L, Jiang WF, Zhou L, Liu X. Early-phase changes of P-wave characteristics after circumferential pulmonary vein isolation. Chin Med J (Engl). 2013;126(14):2607-12.

8. Janin S, Wojcik M, Kuniss M, Berkowitsch A, Erkapic D, Zaltsberg S, et al. Pulmonary vein antrum isolation and terminal part of the P wave. Pacing Clin Electrophysiol. 2010;33(7):784-9.

9. Kizilirmak F, Demir GG, Gokdeniz T, Gunes HM, Cakal B, Guler E, et al. Changes in electrocardiographic P wave parameters after cryoballoon ablation and their association with atrial fibrillation recurrence. Ann Noninvasive Electrocardiol. 2016;21(6):580-7.

10. Buck S, Rienstra M, Maass AH, Nieuwland W, Van Veldhuisen DJ, Van Gelder IC. Cardiac resynchronization therapy in patients with heart failure and atrial fibrillation: importance of new-onset atrial fibrillation and total atrial conduction time. Europace. 2008;10(5):558-65.

11. Van Beeumen K, Houben R, Tavernier R, Ketels S, Duytschaever M. Changes in P-wave area and P-wave duration after circumferential pulmonary vein isolation. Europace. 2010;12(6):798-804.

12. Mugnai G, Chierchia G-B, de Asmundis C, Juliá J, Conte G, Sieira-Moret J, et al. P-wave indices as predictors of atrial fibrillation recurrence after pulmonary vein isolation in normal left atrial size. J Cardiovasc Med. 2016;17(3):194-200. 